\title{
Do immune-related adverse events correlate with response to immune checkpoint inhibitors?
}

\author{
Khalil Saleh ${ }^{1}$, Nadine Khalife-Saleh ${ }^{1} \&$ Hampig Raphael Kourie ${ }^{* 1}$ \\ ${ }^{1}$ Hematology-Oncology Department, Faculty of Medicine, Saint Joseph University of Beirut, Beirut, Lebanon \\ *Author for correspondence: hampig.kourie@hotmail.com
}

\begin{abstract}
"We summarize in this paper the major irAEs of $\mathrm{ICl}$ described in the literature, their association with different agents of $\mathrm{ICl}$ and their correlation with clinical outcome in multiple indications"
\end{abstract}

First draft submitted: 9 December 2018; Accepted for publication: 2 January 2018; Published online: 25 January 2019

\begin{abstract}
Keywords: adverse events $\bullet$ atezolizumab • immune checkpoint inhibitors $\bullet$ immune-related $\bullet$ nivolumab $\bullet$ pem-
\end{abstract} brolizumab

\begin{abstract}
Immune checkpoint inhibitors (ICIs) are changing the natural history of several types of cancer. Ipilimumab which is a monoclonal antibody targeting cytotoxic T lymphocyte-associated antigen 4 is indicated in the treatment of metastatic melanoma. Pembrolizumab and nivolumab, programmed death-1 (PD-1) inhibitors, were approved by the US FDA in the treatment of advanced melanoma, Hodgkin lymphoma, advanced lung cancer, metastatic renal cell carcinoma, advanced urothelial carcinoma, hepatocellular carcinoma, advanced squamous cell carcinoma of the head and neck, metastatic gastric carcinoma positive for PD ligand-1 (PD-L1). Furthermore, atezolizumab, avelumab and durvalumab, anti-PD-L1 are efficacious in the treatment of urothelial carcinoma, Merkel cell carcinoma, metastatic lung cancer and inoperable stage III non-small-cell lung cancer (NSCLC) [1]. Despite the impressive therapeutic benefits, ICI may induce profound immune-related adverse events (irAEs), which can be severe and limit the use of these agents. We summarize in this paper the major irAEs of ICI described in the literature, their association with different agents of ICI and their correlation with clinical outcome in multiple indications.
\end{abstract}

\section{Immune-related adverse effects}

The ICIs have different safety profiles. Ipilimumab was associated with $60-65 \%$ of any grade irAEs when tested in metastatic melanoma and which were mostly grade 1-2 [2]. Grade 3-4 irAEs occurred in 23\% of patients with metastatic melanoma treated with ipilimumab $3 \mathrm{mg} / \mathrm{kg}$ [3]. Skin manifestations were the most common adverse events (43-45\%) followed by gastrointestinal (29-32\%), hepatic and endocrine adverse events (6-8\%). Colitis occurred in $8-22 \%$ of patients receiving ipilimumab while diarrhea was the most common cause of treatment discontinuation [2]. Hypophysitis was observed in 5\% of patients receiving ipilimumab and was more frequent in women than in men [4]. However, PD-1 axis inhibitors were mostly associated with fatigue (24-35\% with nivolumab and pembrolizumab). Diarrhea occurred in $11-13 \%$ of patients treated with anti-PD-1. Cutaneous manifestations such as rash and pruritus were more frequent with pembrolizumab than nivolumab but fewer than ipilimumab [2]. Thyroiditis was the most commonly reported endocrine adverse event, most often presenting as hypothyroidism and more frequently occurred with PD-1 inhibitor than anti-cytotoxic T lymphocyte-associated antigen 4. Pneumonitis has been described more commonly in patients treated with nivolumab compared with other agents [4]. A recent meta-analysis which included clinical trials evaluating pembrolizumab, nivolumab and atezolizumab, compared adverse events of ICI with those of standard chemotherapy. Baxi et al. found that patients treated with ICI were at higher risk of organ-specific irAEs, especially for any grade hypothyroidism (odds ratio [OR]: 6.92; $\mathrm{p}<0.001$ ), pneumonitis (OR: 5.27; $\mathrm{p}<0.001$ ), any grade hypophysitis (OR: 3.38; $\mathrm{p}=0.04$ ) and colitis (OR: 2.88; $\mathrm{p}=0.009$ ) but there was not an increased risk of hepatitis (OR: 3.14; $\mathrm{p}=0.11$ ) [5]. In another meta-analysis which evaluated multiple clinical trials with anti-PD1/PD-L1, it has been demonstrated that the 
development of irAEs was unrelated to the dose of ICI agent. The relative risk of developing any grade of irAE with pembrolizumab at $2 \mathrm{mg} / \mathrm{kg}$ was not statistically different than that with pembrolizumab at $10 \mathrm{mg} / \mathrm{kg}$ (response rate $[\mathrm{RR}]=0.91 ;[0.70-1.17] ; \mathrm{p}=0.46)[6]$.

\section{Melanoma}

The association between irAEs and efficacy of ICI was first described in metastatic melanoma. Data are controversial concerning ipilimumab and PD-1 inhibitors. In a retrospective analysis, the development of irAEs of any grade in patients with metastatic melanoma and who received ipilimumab with or without peptide vaccines was significantly associated with likelihood of response $(\mathrm{p}=0.0004)$. Patients who experienced grade $3-4$ irAEs had a median duration of response of 34 months and those who developed grade 1-2 had a median duration of response of 11 months [7]. However, Weber $e t$ al. found that there was no significant association between irAEs and clinical response $(p=0.45)[8]$. In addition, available data from Memorial Sloan Kettering Cancer Center showed no significant difference in overall survival (OS) and time-to-treatment failure in patients with metastatic melanoma treated with ipilimumab when stratified by the presence or absence of irAEs of any grade [9]. Concerning PD-1 inhibitors, in a pooled analysis of patients with metastatic melanoma receiving nivolumab as monotherapy, objective response rate (ORR) was significantly higher in patients with irAEs. Sanlorenzo et al. reported that development of cutaneous adverse events in patients treated with pembrolizumab had significantly longer progression-free survival (PFS) compared with patients who did not develop cutaneous adverse events [10]. However, a retrospective study concerning patients with advanced melanoma treated with pembrolizumab demonstrated that ORR and disease control rate are similarly observed among patients with irAEs and those who did not develop irAEs $(\mathrm{p}=0.45$ and $\mathrm{p}=0.34$, respectively) [11].

\section{Non-small-cell lung cancer}

In patients with NSCLC, data concerning the association between irAEs and clinical outcomes were more consistent. Haratani et al. reported in a retrospective study, concerning patients treated with nivolumab for NSCLC, that median OS was significantly higher in patients with any grade irAE compared with the group of patients who did not develop irAE (not reached vs 11.1 months; $\mathrm{p}=0.01$ ). The multivariate analysis revealed that irAEs were positively associated with PFS (hazard ratio $[\mathrm{HR}]=0.535 ; \mathrm{p}=0.03)$ and with $\mathrm{OS}(\mathrm{HR}=0.282 ; \mathrm{p}=0.003)$ [12]. In a retrospective cohort of 70 patients with advanced NSCLC treated with nivolumab, the incidence of any grade irAE was significantly higher in responders than nonresponders (76 vs $24 \%$; $<0.001$ ). The median PFS was significantly prolonged in patients with irAEs than those with no-irAE (12.0 vs 3.6 months) [13]. Sato et al. found in a single-institution prospective observational study of 38 patients with NSCLC and treated with nivolumab that patients with irAEs were significantly higher ORR compared with no-irAE patients (63.6 vs 7.4\%; $\mathrm{p}<0.01$ ). Similarly, the median PFS was significantly higher in patients who developed irAEs compared with no-irAEs patients (not reached vs 49 days, $\mathrm{HR}=0.10 ; \mathrm{p}<0.001$ ) [14]. Moreover, Teraoka et al. published the results of a prospective cohort evaluating the association of early irAEs which occur 2-6 weeks after the beginning of treatment and clinical outcome in patients with advanced NSCLC treated with nivolumab. The median PFS in patients who presented irAEs 2 weeks after commencement of nivolumab was significantly higher than median PFS in those who did not develop irAEs (6.4 vs 1.5 months; $\mathrm{p}=0.01$ ). A similar trend was observed in patients with and without 6 weeks irAEs after initiation of nivolumab (4.1 vs 1.5 months; $p=0.06$ ) [15]. These data suggest that early onset of irAEs might be associated with better outcome in patients treated with nivolumab in advanced NSCLC.

\section{Head \& neck squamous cell carcinoma}

In head and neck squamous cell carcinoma, Foster et al. reported at last American Society of Clinical Oncology (ASCO) that irAEs while receiving PD-1 inhibitors are associated with superior ORR, PFS and OS in patients with head and neck cancer unselected for PD-L1 status. The median PFS and OS was 6.9 and 12.5 months, respectively, in patients who developed irAE compared with 2.1 and 6.8 months, respectively, in those who did not develop irAEs $(\mathrm{p}=0.0004$ and $\mathrm{p}=0.0007$, respectively) [16].

\section{Conclusion}

The irAEs are numerous and vary according to ICI agent. It seems that correlation of these irAEs with clinical efficacy is controversial in metastatic melanoma but it is more consistent in NSCLC. Emerging data are supporting 
these findings in head and neck cancer. Further studies with larger numbers of patients and longer follow-up times are needed to validate these findings.

\section{Financial \& competing interests disclosure}

The authors have no relevant affiliations or financial involvement with any organization or entity with a financial interest in or financial conflict with the subject matter or materials discussed in the manuscript. This includes employment, consultancies, honoraria, stock ownership or options, expert testimony, grants or patents received or pending, or royalties.

No writing assistance was utilized in the production of this manuscript.

\section{References}

1. Approvals of FDA-regulated products. www.fda.gov/NewsEvents/ProductsApprovals/

2. Boutros C, Tarhini A, Routier E et al. Safety profiles of anti-CTLA-4 and anti-PD-1 antibodies alone and in combination. Nat. Rev. Clin. Oncol. 13(8), 473-486 (2016)

3. Hodi FS, O’Day SJ, McDermott DF et al. Improved survival with ipilimumab in patients with metastatic melanoma. N. Engl. J. Med. 363(8), 711-723 (2010).

4. Suarez-Almazor ME, Kim ST, Abdel-Wahab N, Diab A. Review: immune-related adverse events with use of checkpoint inhibitors for immunotherapy of cancer. Arthritis Rheumatol. Hoboken, NJ, USA 69(4), 687-699 (2017).

5. Baxi S, Yang A, Gennarelli RL et al. Immune-related adverse events for anti-PD-1 and anti-PD-L1 drugs: systematic review and meta-analysis. BMJ 360, k793 (2018).

6. Wang P-F, Chen Y, Song S-Y et al. Immune-related adverse events associated with anti-PD-1/PD-L1 treatment for malignancies: a meta-analysis. Front. Pharmacol. 8, 730 (2017).

7. Downey SG, Klapper JA, Smith FO et al. Prognostic factors related to clinical response in patients with metastatic melanoma treated by CTL-associated antigen-4 blockade. Clin. Cancer Res. Off. J. Am. Assoc. Cancer Res. 13(22 Pt 1), 6681-6688 (2007).

8. Weber JS, O’Day S, Urba W et al. Phase I/II study of ipilimumab for patients with metastatic melanoma. J. Clin. Oncol. 26(36), 5950-5956 (2008).

9. Horvat TZ, Adel NG, Dang T-O et al. Immune-related adverse events, need for systemic immunosuppression, and effects on survival and time to treatment failure in patients with melanoma treated with ipilimumab at memorial sloan kettering cancer center. J. Clin. Oncol. 33(28), 3193-3198 (2015).

10. Sanlorenzo M, Vujic I, Daud A et al. Pembrolizumab cutaneous adverse events and their association with disease progression. JAMA Dermatol. 151(11), 1206-1212 (2015).

11. Grimaldi AM, Simeone E, Festino L et al. Correlation between immune-related adverse events and response to pembrolizumab in advanced melanoma patients. J. Immunother. Cancer 3(Suppl. 2), P186 (2015).

12. Haratani $\mathrm{K}$, Hayashi $\mathrm{H}$, Chiba $\mathrm{Y}$ et al. Association of immune-related adverse events with nivolumab efficacy in non-small-cell lung cancer. JAMA Oncol. 4(3), 374-378 (2018).

13. Toi Y, Sugawara S, Kawashima Y et al. Association ofimmune-related adverse events with clinical benefit in patients with advanced non-small-cell lung cancer treated with nivolumab. Oncologist 1358-1365 23 (2018).

14. Sato K, Akamatsu H, Murakami E et al. Correlation between immune-related adverse events and efficacy in non-small cell lung cancer treated with nivolumab. Lung Cancer Amsterdam, The Netherlands 115, 71-74 (2018).

15. Teraoka S, Fujimoto D, Morimoto T et al. Early immune-related adverse events and association with outcome in advanced non-small cell lung cancer patients treated with nivolumab: a prospective cohort study. J. Thorac. Oncol. 12(12), 1798-1805 (2017).

16. Foster CC, Kochanny S, Khattri A et al. Association of immune-related adverse events (irAEs) with improved response, progression-free survival, and overall survival for patients with metastatic head and neck cancer receiving anti-PD-1 therapy. J. Clin.

Oncol. 36(15_suppl.), 6014-6014 (2018). 